(Supporting Information)

\title{
Interface-stabilized Layered Lithium Ni-rich Oxide Cathode via Surface Functionalization with
} Titanium Silicate

Giseung Lee, ${ }^{+\$}$ Kwangeun Jung, ${ }^{+\$}$ Yongho Lee, Jeonghan Kim, ${ }^{+, *}$ Taeeun Yim ${ }^{+, *}$

Department of Chemistry, Incheon National University, 119 Academy-ro, Yeonsu-gu, Incheon 22012, Republic of Korea

Cathode Material R\&D Group, POSCO CHEMICAL, 87, Chemdangieop 1-ro, Sandong-myeon, Gumi-si, Gyeongsangbuk-do, 39171 Republic of Korea 
*Corresponding authors: yte0102@inu.ac.kr (T. Yim) and jeonghan.kim@poscochemical.com (J.

Kim)

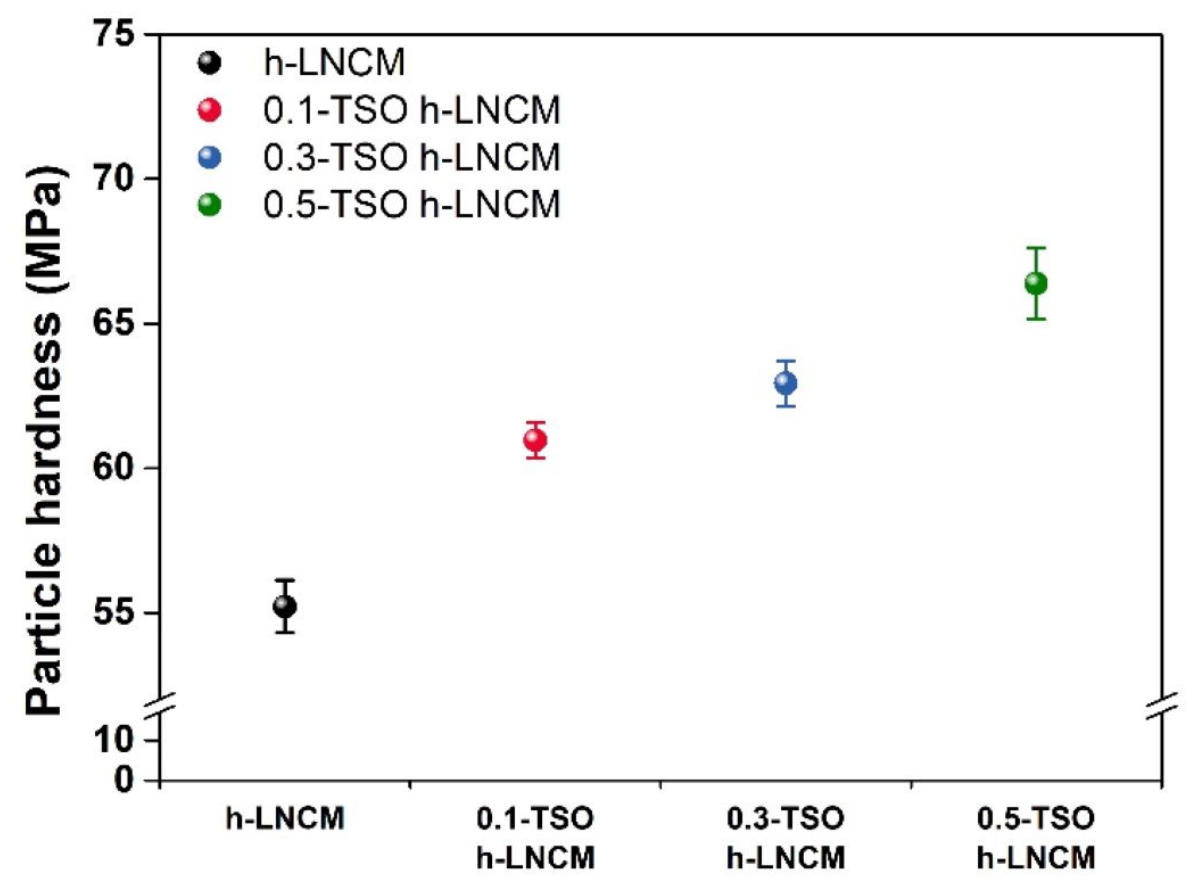

Figure S1. Particle hardness of h-LNCM cathode materials after electrolyte immersing (hLNCM (black), 0.1-TSO h-LNCM (red), 0.3-TSO h-LNCM (blue) and 0.5-TSO h-LNCM (green)). 
(a)

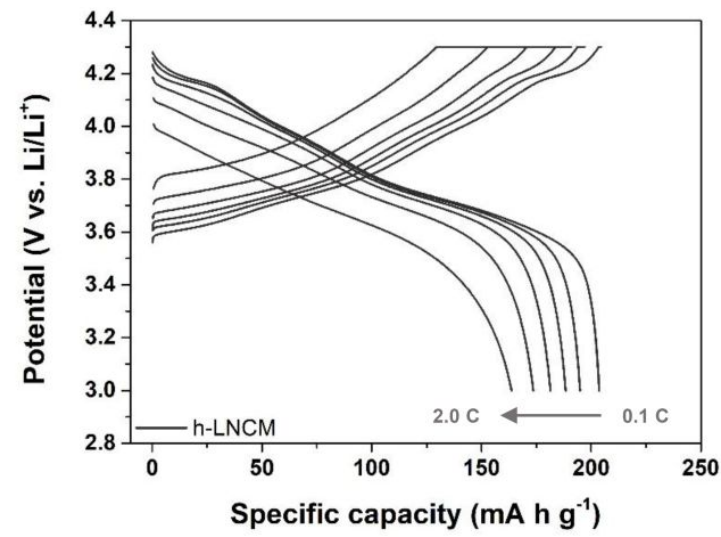

(b)

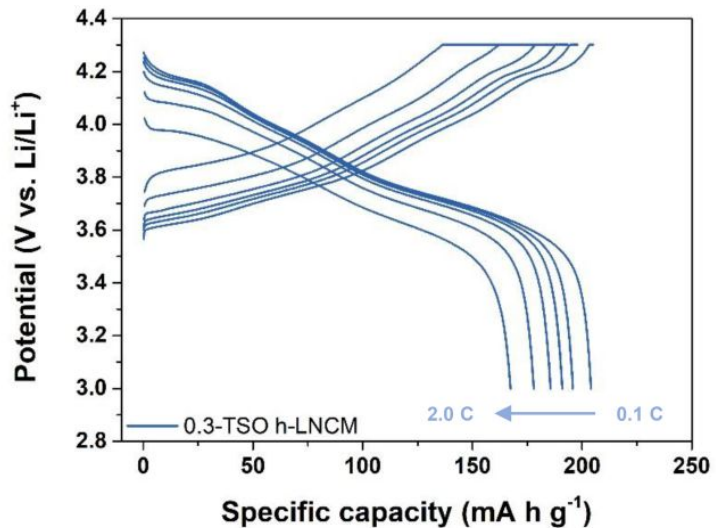

Figure S2. Rate capabilities of the cell cycled with (a) h-LNCM cathode (black) and (b) 0.3TSO h-LNCM cathode (blue). 
(a)

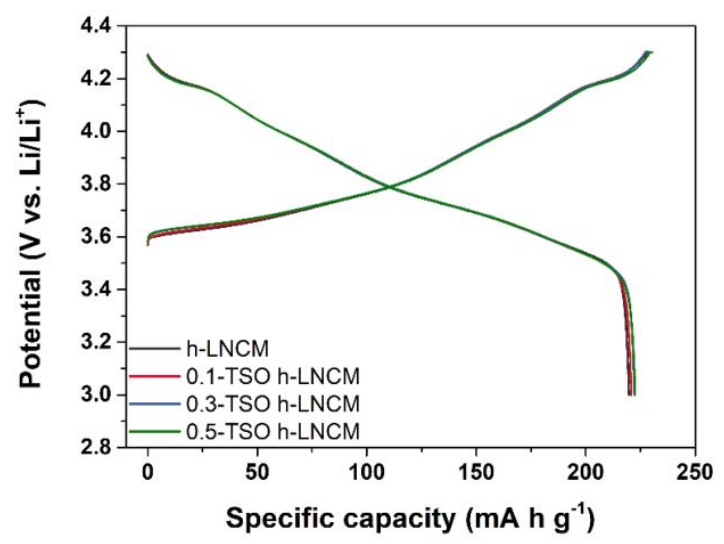

(b)

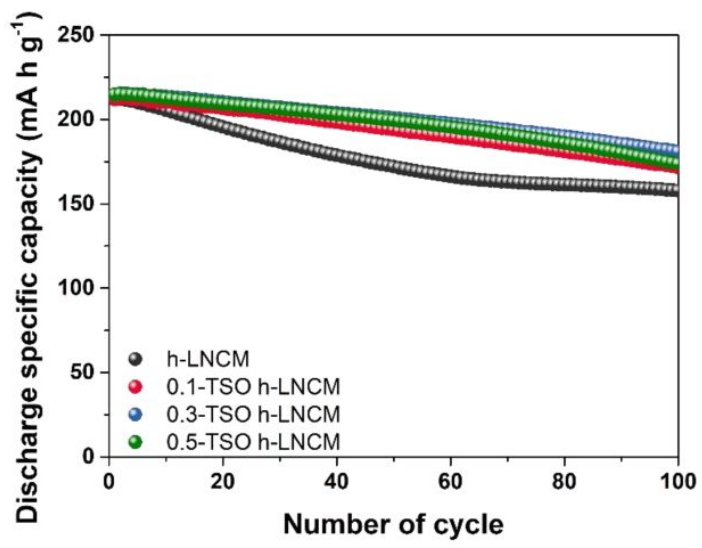

Figure S3. (a) Potential profiles and (b) cycling performance of the cells cycled with h-LNCM cathode (black), 0.1-TSO h-LNCM cathode (red), 0.3-TSO h-LNCM cathode (blue), and 0.5TSO h-LNCM cathode (green) at high temperature. 